\title{
Effects of Chemical Sympathectomy on Lipoprotein Lipase Activities in Peripheral Tissues of Rats Fed High Fat Diets Consisting of Different Fats
}

\author{
Tatsuhiro Matsuo, ${ }^{*}$ Hiroshi Sumida, and Masashige Suzuki ${ }^{1}$ \\ Division of Nutrition and Biochemistry, Sanyo Womem's College, \\ Hatsukaichi 738, Japan \\ ${ }^{1}$ Institute of Health and Sport Sciences, University of Tsukuba, \\ Tsukuba 305, Japan
}

(Received February 24, 1995)

\begin{abstract}
Summary We have previously reported that the feeding of a beef tallow diet gives lower lipoprotein lipase activities in the interscapuler brown adipose tissue, heart and soleus muscle compared with feeding of a safflower oil diet. We have also suggested that sympathetic activities (norepinephrine turnover rates and $\beta$-adrenergic receptor bindings) in these peripheral tissues were lower in the beef tallow diet group. To confirm the effects of dietary fats on the lipoprotein lipase activities in these tissues related to sympathetic activities, rats were chemically sympathectomized by treatment with 6-hydroxydopamine. The sympathectomized rats and control rats were meal-fed with a beef tallow diet or a safflower oil diet for 8 weeks. Sympathectomy abolished the differences in lipoprotein lipase activities in the interscapuler brown adipose tissue, heart and soleus muscle between two dietary groups. The percentage of body fat was increased by sympathectomy, resulting in no difference between the beef tallow diet group and the safflower oil diet group. These results suggest that intake of the beef tallow diet decreased lipoprotein lipase activities by reducing the sympathetic activities.
\end{abstract}

Key Words lipoprotein lipase, sympathectomy, brown adipose tissue, heart, soleus muscle, beef tallow, safflower oil, rat

Lipoprotein lipase (LPL; EC 3.1.1.34), a hydrolytic enzyme produced by many tissues, is rate-limiting for the removal of lipoprotein triacylglycerols from the circulation (1-3). LPL has its physiological site of action at the luminal surface of capillary endothelial cells where the enzyme hydrolyses the triacylglycerol component of circulating lipoprotein particles, chylomicrons and very low density lipoproteins, to provide free fatty acids and 2-monoacylglycerol for tissue utilization (1-3). LPL plays a primary role in triacylglycerol metabolism and as well as

\footnotetext{
* To whom correspondence should be addressed.
} 
an important role in certain metabolic disorders, including obesity (4-6).

We have recently demonstrated that when rats were meal-fed isoenergetic diets (45\% of energy as fat) based on beef tallow (rich in saturated and monounsaturated fatty acids) or safflower oil (rich in $n-6$ polyunsaturated fatty acid) for 8-16 weeks, LPL activities in the interscapular brown adipose tissue (IBAT), heart and soleus muscle were lower in rats fed a beef tallow diet than in rats fed a safflower oil diet, and consequently greater body fat was accumulated in the former $(7,8)$. On the other hand, since LPL activities in IBAT, heart and soleus muscle are increased by $\beta$-adrenergic stimulation (9-12), we examined the effect of the beef tallow diet intake on sympathetic activities in the IBAT, heart and soleus muscle compared with the safflower oil diet intake. It was clearly suggested in the previous study that $\beta$-adrenergic receptor bindings and norepinephrine (NE) turnover rates in the IBAT, heart and soleus muscle were decreased by the intake of a beef tallow diet resulting from the decrease of membrane fluidities of those organs (13). However, a question still remains whether or not the reductions of tissue LPL activities in the beef tallow diet group are independent of the decreases of sympathetic activities.

6-Hydroxydopamine (6-OHDA), a false neurotransmitter, is known to produce chronic chemical sympathectomy, and it was generally used to examine the role of the sympathetic nervous system in the control of metabolic and hormonal responses $(14,15)$. In the present study, we directly investigated the relationship between LPL activities and sympathetic activities in the IBAT, heart and soleus muscle using chemically sympathectomized rats.

\section{EXPERIMENTAL}

All procedures involving animals were approved by the Experimental Animal Care Committee of the University of Tsukuba.

Animals. Male and female Sprague-Dawley rats (10 weeks old) were obtained from CLEA Japan. Rats were fed with CE-2, a commercial rodent diet (CLEA Japan) and given free access to water. They were mated at 12 weeks of age, and 36 male pups obtained were used in this experiment. The sympathectomy was performed according to the modification as described previously $(16,17)$. Nineteen pups were treated by i.p. injection with $0.6 \mathrm{mmol} / \mathrm{kg}$ 6-hydroxydopamine hydrobromide (6-OHDA, Aldrich, Milwaukee, WI) dissolved in saline (final concentration; $0.6 \mathrm{M}$ ) everyday for the first week and on day 14 and day 21 after birth. The remaining rats were treated with saline as a control. The validity of this protocol to produce effective sympathectomy accessed by both functional and biochemical means has been reported previously $(14,15)$.

After weaning ( 3 weeks of age), control and sympathectomized rats were fed nonpurified diet and water with free access through 5 weeks of age. At 5 weeks of age, 5 rats in each group were killed by decapitation. NE contents of the IBAT, heart and soleus muscle were analyzed in the rats described below, and it was 
confirmed that the NE contents were decreased to $3-10 \%$ of the control rats by the 6-OHDA treatment.

Diets and experimental design. Then control $(n=12)$ and sympathectomized rats were randomly divided into two subgroups: the safflower oil diet and beef tallow diet groups. The compositions of both diets have been described previously (7). Both diets provided 45, 35 and 20\% of energy as fat, carbohydrate and protein, respectively. The metabolizable energy was $19.7 \mathrm{~kJ} / \mathrm{g}$ for the safflower oil diet and $18.4 \mathrm{~kJ} / \mathrm{g}$ for the beef tallow diet. The fatty acid compositions of safflower oil and beef tallow have been described previously (7); beef tallow consisted of $44 \%$ oleic, 27\% palmitic and 18\% stearic acids and safflower oil consisted of $79 \%$ linoleic acids.

The animals were individually caged at $22 \pm 2{ }^{\circ} \mathrm{C}$, with light from $0700 \mathrm{~h}-1900$ $\mathrm{h}$. Each group of rats was meal-fed the diet at $0800-0900 \mathrm{~h}$ and $2000-2100 \mathrm{~h}$, and was given free access to water for 8 weeks. Food consumption of the control rats was adjusted by the meal-feeding method at the same metabolizable energy between the safflower oil diet group and the beef tallow diet group (Table 1), and was approximately the maximal amount of diet that rats could consume under the meal-feeding conditions. Food consumption of sympathectomized rats was also adjusted between dietary groups as described above. Approximately $80 \%$ of energy for the control rats were basically provided to the sympathectomized rats (Table 1). It has been confirmed that sympathectomized rats can consume this amount of food under meal-feeding conditions.

At the end of 8 weeks feeding on the experimental diet, the rats were killed by decapitation at $0800 \mathrm{~h}$. Blood was collected to obtain serum and IBAT, heart and soleus muscle were quickly removed, weighed, and stored at $-80^{\circ} \mathrm{C}$ until analysis. Carcass samples were obtained by removing the head, liver, lungs, kidneys, spleen, testes, pancreas and digestive tracts and were stored at $-20^{\circ} \mathrm{C}$ until analysis of carcass composition.

Table 1. Food consumption of rats fed both experimental diets. ${ }^{1}$

\begin{tabular}{ccc}
\hline \multirow{2}{*}{$\begin{array}{c}\text { Experimental period } \\
\text { (week) }\end{array}$} & \multicolumn{2}{c}{ Food consumption $(\mathrm{MJ} /$ week) } \\
\cline { 2 - 3 } & Control & 6-OHDA \\
\hline 1 & 1.99 & 1.51 \\
2 & 2.45 & 2.03 \\
3 & 2.51 & 2.10 \\
4 & 2.66 & 2.24 \\
5 & 2.70 & 2.34 \\
6 & 2.45 & 1.96 \\
7 & 2.44 & 1.92 \\
8 & 2.32 & 1.83 \\
Total & 19.5 & 15.9 \\
\hline
\end{tabular}

${ }^{1}$ Values were calculated as the metabolizable energy of the diets. 
Serum and carcass analyses. Serum glucose and triacylglycerol were measured enzymatically using kits, glucose C-II Test and Triglyceride G Test, respectively, purchased from Wako Pure Chemical Industries (Osaka). Serum insulin was determined by an enzyme immunoassay using a kit (insulin EIA kit) purchased from Sanko Junyaku Co., Ltd. (Tokyo). Carcass fat and protein were analyzed by the method of Mickelsen and Anderson (18).

$N E$ contents in various tissues. NE contents in the IBAT, heart and soleus muscle were assayed by high-performance liquid chromatography with electrochemical detection (Shimadzu Corp., Kyoto, Model LC-6A) as modified by Refshauge et al. (19).

LPL activities of various tissues. LPL activities of the IBAT, heart and soleus muscle were measured by the methods described our previous paper (13). Briefly, the tissues were prepared by the method of Mori et al. (20). The substrate for LPL was prepared according to the method of Nilsson-Ehle and Schotz (21), but using unlabeled triolein. The LPL activity assay was performed by incubation of the extract with the substrate at $37^{\circ} \mathrm{C}$ for $30 \mathrm{~min}$. The free fatty acids released during the incubation were measured enzymatically as described above. One unit of LPL activity is defined as catalyzing the release of $1 \mu \mathrm{mol}$ free fatty acid per hour.

Statistical analysis. Statistical differences in final body weight, tissue weights, carcass composition, serum components, NE contents and LPL activities were analyzed by two-way ANOVA and Scheffe's test (22). Statistical differences in initial body weight were analyzed by Student's $t$-test.

\section{RESULTS}

\section{Body weight, tissue weights and carcass composition}

The dietary regimens were initiated at 5 weeks of age in both the sympathectomized and control rats. The initial body weight at 5 weeks of age was lower in the sympathectomized rats than in the control rats (Table 2). During the experimental period, the sympathectomized rats showed a lower growth rate compared to the control rats (Table 2). Neither dietary fats nor sympathectomy affected the IBAT weight (Table 2). The weights of heart and soleus muscle were lower in the sympathectomized rats than in the control rats but were not significantly different between the two dietary groups (Table 2). The weight of abdominal adipose tissues were lower in the sympathectomized rats, however the percentage of those tissues to final body weight was not affected by sympathectomy (range of mean values: $5.6-6.2 \%$ ). In control rats, the carcass fat content was significantly greater $(p<0.05)$ in the beef tallow diet group than in the safflower oil diet group (Table 2). On the other hand, the percentage of carcass fat was increased by sympathectomy in both dietary groups, with no difference between them (Table 2). The percentage of carcass protein was not affected by either dietary fats or sympathectomy (Table 2). 
Table 2. Effects of the dietary fats on body weight, abdominal adipose tissue weight and carcass composition in synpathectomized rats. ${ }^{1}$

\begin{tabular}{|c|c|c|c|c|}
\hline & \multicolumn{2}{|c|}{ Control } & \multicolumn{2}{|c|}{ 6-OHDA } \\
\hline & Safflower oil & Beef tallow & Safflower oil & Beef tallow \\
\hline \multicolumn{5}{|l|}{ Body weight } \\
\hline Initial (g) & \multicolumn{2}{|c|}{$145 \pm 3$} & \multicolumn{2}{|c|}{$114 \pm 2^{\dagger}$} \\
\hline Final $(\mathrm{g})$ & $430 \pm 2$ & $425 \pm 3$ & $326 \pm 3^{\S}$ & $321 \pm 2^{\S}$ \\
\hline \multicolumn{5}{|l|}{ Tissue weights } \\
\hline BAT (mg) & $550 \pm 39$ & $483 \pm 35$ & $556 \pm 22$ & $497 \pm 59$ \\
\hline Heart (mg) & $1,086 \pm 27$ & $1,121 \pm 29$ & $838 \pm 43^{\S}$ & $828 \pm 26^{8}$ \\
\hline Soleus muscle (mg) & $331 \pm 14$ & $320 \pm 7$ & $264 \pm 10^{\S}$ & $247 \pm 9^{\S}$ \\
\hline WAT $(\mathrm{g})$ & $24 \pm 2$ & $27 \pm 2$ & $18 \pm 2^{\S}$ & $20 \pm 2^{\S}$ \\
\hline Carcass weight $(\mathrm{g})$ & $298 \pm 3$ & $291 \pm 4$ & $226 \pm 2^{\S}$ & $222 \pm 3^{\S}$ \\
\hline \multirow{2}{*}{$\begin{aligned} \text { Carcass fat } & (\mathrm{g}) \\
& (\%)\end{aligned}$} & $34 \pm 1$ & $43 \pm 1^{*}$ & $47 \pm 2^{8}$ & $46 \pm 1$ \\
\hline & $11 \pm 1$ & $15 \pm 1^{*}$ & $21 \pm 1^{\S}$ & $21 \pm 1^{\S}$ \\
\hline Carcass protein (g) & $66 \pm 1$ & $67 \pm 2$ & $48 \pm 2^{\S}$ & $49 \pm 1^{\S}$ \\
\hline$(\%)$ & $22 \pm 1$ & $23 \pm 1$ & $22 \pm 1$ & $22 \pm 1$ \\
\hline
\end{tabular}

${ }^{1}$ Values are means \pm SE for $5-7$ rats. ${ }^{\dagger}$ Statistically significant difference $(p<0.01)$ from the control (Student's $t$-test). * Statistically significant difference $(p<0.05)$ from the safflower oil diet group. ${ }^{\S}$ Statistically significance $(p<0.05)$ from the control (two-way ANOVA and Scheffe's test). BAT, brown adipose tissue; WAT, abdominal white adipose tissues (epididymal, perirenal and mesenteric).

Table 3. Effect of the dietary fats on serum glucose, free fatty acids and triacylglycerol levels in sympathectomized rats. ${ }^{1}$

\begin{tabular}{lccccc}
\hline & \multicolumn{2}{c}{ Control } & & \multicolumn{2}{c}{ 6-OHDA } \\
\cline { 2 - 3 } \cline { 5 - 6 } & Safflower oil & Beef tallow & & Safflower oil & Beef tallow \\
\hline Glucose $(\mathrm{mg} / \mathrm{dl})$ & $119 \pm 3$ & $110 \pm 4$ & & $110 \pm 4$ & $110 \pm 3$ \\
Insulin $(\mu \mathrm{U} / \mathrm{ml})$ & $14 \pm 2$ & $26 \pm 2^{*}$ & & $33 \pm 3^{\S}$ & $32 \pm 2^{\S}$ \\
Triacylglycerol $(\mathrm{mg} / \mathrm{dl})$ & $27 \pm 2$ & $60 \pm 2^{*}$ & & $52 \pm 4^{\S}$ & $64 \pm 6$ \\
\hline
\end{tabular}

${ }^{1}$ Values are means \pm SE for $5-7$ rats. $*$ Statistically significant difference $(p<0.05)$ from the safflower oil diet group. ${ }^{\S}$ Statistically significant difference $(p<0.05)$ from the control (two-way ANOVA and Sheffe's test).

Serum glucose, free fatty acids and triacylglycerol concentrations

Neither dietary fats nor sympathectomy affected the level of serum glucose (Table 3). The sympathectomy elevated the serum levels of insulin and triacylglycerol (Table 3). The serum insulin level of control rats was significantly higher in the beef tallow diet group $(p<0.05)$, whereas no dietary effect was observed on the levels in sympathectomized rats (Table 3 ). The serum triacylglycerol level was significantly higher $(p<0.05)$ in the beef tallow diet group, however the difference between the two diet groups was not significant in the sympathectomized rats (Table 3 ). 
$N E$ content in various tissues

In the control rats, the NE content in IBAT, heart and soleus muscle was lower in the beef tallow diet group (Fig. 1), and these results were similar to those previously reported. The tissue NE content was decreased to $4-15 \%$ of the control rats in IBAT, heart and soleus muscle by the 6-OHDA treatment.

\section{LPL activities in various tissues}

In the control rats, LPL activities in the IBAT, heart and soleus muscle were lower $(p<0.05)$ in the beef tallow diet group than in the safflower oil diet group (Fig. 2). Sympathectomy decreases LPL activities in various tissues, with no
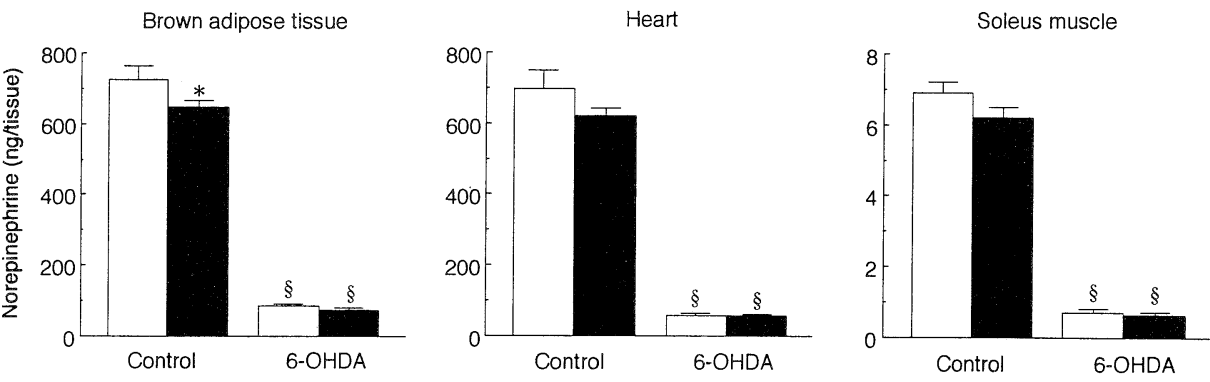

Fig. 1. Norepinephrine content in the IBAT, heart and soleus muscle in rats fed the beef tallow diet or the safflower oil diet. Values are means and SE for 5-7 rats. ${ }^{\S}$ Statistically significant difference from the control. Differences with $p<0.05$ (ANOVA and Scheffe's test) were considered to be significant.
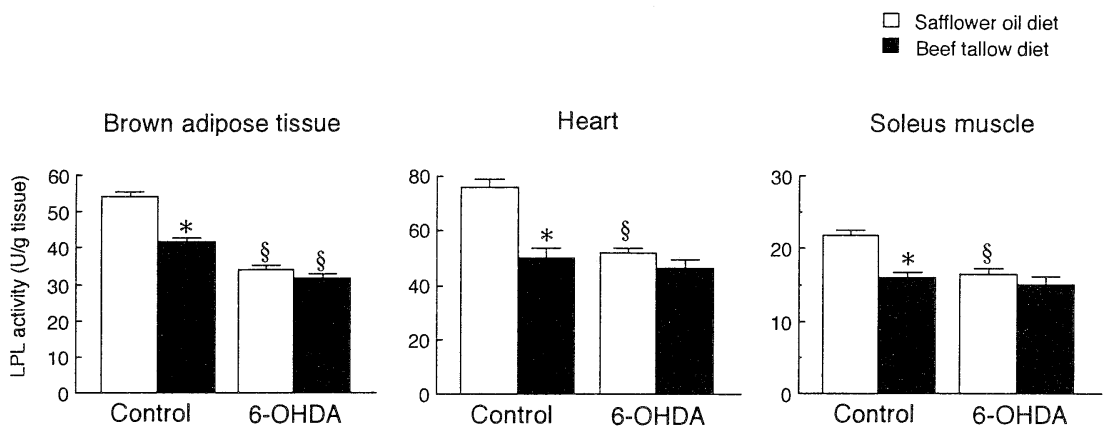

Fig. 2. Lipoprotein lipase activity in the IBAT, heart and soleus muscle in rats fed the beef tallow diet or the safflower oil diet. Values are means and SE for 5-7 rats. * Statistically significant difference from the safflower oil diet group. ${ }^{\S}$ Statistically significant difference from the control. Differences with $p<0.05$ (ANOVA and Scheffe's test) were considered to be significant. 
difference between the two dietary groups (Fig. 2). The reduction rates of LPL activity by sympathectomy was greater in the safflower oil diet group (37.6 vs. $22.4 \%$ for IBAT, 31.4 vs. $8.0 \%$ for heart, 24.8 vs. $6.3 \%$ for soleus muscle).

\section{DISCUSSION}

We clearly showed that sympathectomy by 6-OHDA treatment dramatically reduced LPL activities in the IBAT, heart and soleus muscle, and it suggested that LPL activities in these tissues were regulated by the sympathetic nervous system.

It is well known that 6-OHDA destroys the whole neuron if administered to new-born animals, and it provides a permanent sympathectomy $(14,15)$. Therefore, 6-OHDA treatment is an established method for sympathectomy. Furthermore, 6-OHDA treatment does not affect any physiological characteristics other than the sympathetic nervous system $(14,15)$. Tissue NE levels adequately reflect sympathetic innervation of the IBAT, heart and soleus muscle. Since the NE content in the IBAT, heart and soleus muscle decreased to $4-15 \%$ of the control rats, we confirmed that sympathectomy was complete in this experiment. In addition, none of the rats treated with 6-OHDA died.

Other studies have shown that the adrenal medulla is not affected by 6-OHDA and that it continues to produce and secrete catecholamines in an effort to compensate for the absence of an intact peripheral sympathetic nervous system (14). However, the NE and epinephrine levels in the adrenal glands were not affected by either dietary fats or sympathectomy (ranges of mean values: 2.5-2.9 $\mu \mathrm{g} /$ tissue for NE and 3.7-4.2 $\mu \mathrm{g} /$ tissue for epinephrine) in this experiment. These results suggest that catecholamines from the adrenal glands are less concerned with LPL activities in the IBAT, heart and soleus muscle.

The lower levels of LPL activities in the IBAT, heart and soleus muscle of the sympathectomized rats suggest that blood triacylglycerol was taken into these tissues at a lower rate in the sympathectomized rats than in the control rats; this view is consistent with the higher level of serum triacylglycerol in the former than the latter. On the other hand, it may be thought that the hyperglycemia observed in the sympathectomized rats was caused by the increase of lipogenesis in the liver and adipose tissues, and the release of triacylglycerol from the liver (23). However, since we used a high-fat and low-carbohydrate diet (45, 35 and 20\% of energy as fat, carbohydrate and protein, respectively) in this experiment, the contribution of lipogenesis in the liver and white adipose tissues to the higher triacylglycerol level in the sympathectomized rats appears to be minor.

The sympathectomized rats had lower body weights than the control rats and gained less weight during fat diet-feeding because the former had lower food intake than the latter. On the other hand, the sympathectomized rats had a greater percentage of carcass fat than the control rats, suggesting that the lower sympathetic activity promoted body fat accumulation. Since the IBAT, heart and skeletal muscles play important roles in energy expenditure (e.g., basal metabolism, diet- 
induced thermogenesis) and fat oxidation $(24,25)$, the lower levels of LPL activities in these tissues may decrease energy expenditure. Our previous study showed that intake of a beef tallow diet decreases LPL activities in the IBAT, heart and soleus muscle compared with intake of a safflower oil diet in rats, and suggested that this effect of a beef tallow diet is at least in part due to lower sympathetic activity, NE turnover and $\beta$-adrenergic receptor bindings in the IBAT, heart and soleus muscle (13). These points strongly suggest that intake of the beef tallow diet produces larger body fat accumulation, probably due to the lower LPL activities resulting from the lower sympathetic activity.

LPL activity is regulated by nutritional and hormonal factors $(1,26)$. Serum insulin increases LPL activity in the adipose tissue but inhibits that in the heart with meal ingestion (26) by a mechanism which does not involve the sympathetic nervous system in these tissues (possibly, post-transcriptional regulation of the LPL gene). In this study, the serum insulin level increased in the sympathectomized rats, with no difference between the two diet groups. This result suggests that sympathectomy promotes hormone release from pancreatic $\beta$-cells. We previously reported that the NE turnover rate in the pancreas was lower in the beef tallow diet group than in the safflower oil diet group, in accordance with the higher serum insulin level in the former (8). These results suggest that the higher serum insulin level in the beef tallow diet group was caused by reducing sympathetic acitvity in the pancreas.

In conclusion, the present study demonstrated that the reduction of sympathetic activity causes a decrease in the LPL activity in the IBAT, heart and soleus muscle, and suggested that beef tallow diet decreases LPL activity by reducing the sympathetic activity in these tissues.

\section{REFERENCES}

1) Braun, J. E. A., and Severson, D. L. (1992): Regulation of synthesis, processing and translocation of lipoprotein lipase. Biochem. J., 287, 337-347.

2) Engelber, H. (1966): Mechanisms involved in the reduction of serum triglycerides in man upon adding unsaturated fats to the normal diet. Metabolism, 15, 796-807.

3) Bagdade, J. D., Hazzard, W. R., and Carlin, J. (1970): Effect of unsaturated dietary fat on plasma lipoprotein lipase activity in normal and hyperlipidemic states. Metabolism, 19, 1020-1024.

4) Eckel, R. H. (1987): Adipose tissue lipoprotein lipase, in Lipoprotein Lipase, ed. by Borensztajn, J., Evener, Chicago, pp. 79-132.

5) Cleary, M. P., Vasselli, J. R., and Greenwood, M. R. C. (1980): Development of obesity in Zucker obese (fa/fa) rats in absence of hyperphagia. Am. J. Physiol., 238, E282-E294.

6) Eckel, R. H., and Yost, T. J. (1987): Weight reduction increases adipose tissue lipoprotein lipase responsivenss in obese women. J. Clin. Invest., 80, 992-997.

7) Shimomura, Y., Tamura, T., and Suzuki, M. (1990): Less body fat accumulation in rats fed a safflower oil diet than in rats fed a beef tallow diet. J. Nutr., 120, 1291-1296. 
8) Matsuo, T., Shimomiara, Y., Saitoh, S., Tokuyama, K., Takeuchi, H., and Suzuki, M. (1995): Sympathetic activity is lower in rats fed a beef tallow diet than in rats fed a safflower oil diet. Metabolism, in press.

9) Radomski, M. W., and Orme, T. (1971): Response of lipoprotein lipase in various tissues to cold exposure. Am. J. Physiol., 220, 1852-1856.

10) Miller, W. C., Gorski, J., Oscai, L. B., and Palmer, W. K. (1989): Epinephrine activation of heparin-nonreleasable lipoprotein lipase in 3 skeletal muscle fiber types of the rat. Biochem. Biophys. Res. Commun., 164, 615-619.

11) Carneheim, C., Nedergaard, J., and Cannon, B. (1984): $\beta$-Adrenergic stimulation of lipoprotein lipase in rat brown adipose tissue during acclimation to cold. Am. J. Physiol., 246, E327-E333.

12) Carneheim, C., Nedergaard, J., and Cannon, B. (1988): Cold-induced $\beta$-adrenergic recruitment of lipoprotein lipase in brown fat is due to increased transcription. Am. J. Physiol., 254, E155-E161.

13) Matsuo, T., and Suzuki, M. (1994): Beef tallow diet decreases lipoprotein lipase activities in brown adipose tissue, heart and soleus muscle by reducing sympathetic activities in rats. J. Nutr. Sci. Vitaminol., 40, 569-581.

14) Thoenen, H., and Tranzer, J. P. (1973): The pharmacology of 6-hydroxydopamine. Annu. Rev. Pharmacol., 113, 169-180.

15) Depocas, F., Foster, D. O., Zaror-Behrens, G., Lacelle, S., and Nadeau, B. (1984): Recovery of function in sympathetic nerves of interscapular brown adipose tissue of rats treated with 6-hydroxydopamine. Can. J. Physiol. Pharmacol., 62, 1327-1332.

16) Desautels, M., and Dulos, R. A. (1990): Effect of neonatal sympathectomy on brown fat development and susceptibility to high fat diet induced obesity in mice. Can. J. Physiol. Pharmacol., 69, 1868-1874.

17) Thomas, G. D., O'Hargan, K. P., and Zambraski, E. J. (1973): Chemical sympathectomy alters the development of hypertension in miniature swine. Hypertension, 17, 357-362.

18) Mickelsen, O., Anderson, A. A. (1959): A method for preparing intact animals for carcass analysis. J. Lab. Clin. Med., 53, 282-290.

19) Refshauge, C., Kissinger, P. T., Dreiling, R., Blank, L., Freeman, R., and Adams, R. N. (1973): New high performance liquid chromatographic analysis of brain catecholamines. Life Sci., 14, 311-322.

20) Mori, N., Murase, T., Yamada, N., Arajawa, N., and Takaku, F. (1984): Wide variations of plasma triglyceride concentrations in guinea pigs. Lipids, 19, 978-981.

21) Nilsson-Ehle, P., and Schotz, M. C. (1976): A stable, radioactive substrate emulsion for assay of lipoprotein lipase. J. Lipid Res., 17, 536-541.

22) Scheffe, H. (1959): The Analysis of Variance. Wiley, New York.

23) Matsuo, T., and Suzuki, M. (1994): Safflower oil diet decreases hepatic acetyl CoA-carboxylase activity by increasing $\alpha_{1}$-adrenergic stimulation in rats. J. Clin. Biochem. Nutr., 17, 179-188.

24) Danforth, E., Jr. (1992): Regulation of energy expenditure, in Obesty: Basic Concepts and Clinical Aspects, ed. by Belfiore, F., Jeanrenaud, B., and Papalia, D., Karger, Basel, pp. 61-77.

25) Rothwell, N. J., and Stock, M. J. (1983): Diet-induced thermogenesis, in Mammalian Thermogenesis, ed. by Girardier, L., and Stock, M. J., Chapman and Hall, London, pp. 
208-233.

26) Nilsson-Ehle, P., Garfinkel, A. S., and Schotz, M. C. (1980): Lipolytic enzymes and plasma lipoprotein metabolism. Annu. Rev. Biochem., 49, 667-693. 